\title{
Response to the comment on Schuh et al.: Angular correction and complications of proximal first metatarsal osteotomies for hallux valgus deformity
}

\author{
Reinhard Schuh • Madeleine Willegger • \\ Johannes Holinka $\cdot$ Robin Ristl $\cdot$ Reinhard Windhager • \\ Axel H. Wanivenhaus
}

Received: 12 November 2013 / Accepted: 13 November 2013/Published online: 30 November 2013

(C) Springer-Verlag Berlin Heidelberg 2013

Dear Prof. Pecina,

We greatly appreciate the comments offered by Dr. He and Dr. Peng regarding the recent publication "Angular correction and complications of proximal first metatarsal osteotomies for hallux valgus deformity" [1]. To the raised claims I would like to comment as follows:

1. He and Peng detected six studies included in our metaanalysis to be suspicious for double-counting patients [2-7]. I reviewed these publications once again and concluded that for the publications from Coughlin et al. [2, 3] this is not the case. Coughlin's publication from 2005 entitled "Proximal metatarsal osteotomy and distal soft tissue reconstruction as treatment for hallux valgus deformity" was a review article which contains results of 27 juvenile patients (33 feet) with hallux valgus. These patients were operated over an 11-year period and were part of the patient cohort from Coughlin's study concerning juvenile hallux valgus published in 1995 [8]. The 103 patients (122 feet) treated with proximal crescentic osteotomy whose results were presented in 2007 [3] were all operated between September 1999 and May 2002. Therefore, the authors did not include overlapping patient data from Coughlin et al. Sammarco et al. reported results of the proximal chevron osteotomy in 1993 and in 1998 [4,

R. Schuh $(\bowtie) \cdot M$. Willegger $\cdot$ J. Holinka $\cdot$ R. Windhager $\cdot$

A. H. Wanivenhaus

Department of Orthopaedics, Medical University of Vienna,

Waehringer Guertel 18-20, 1090 Vienna, Austria

e-mail: reinhard.schuh@meduniwien.ac.at

\section{R. Ristl}

Section for Medical Statistics, Center for Medical Statistics, Informatics, and Intelligent Systems, Medical University of Vienna, Vienna, Austria
5]. The first study comprised 43 patients (51 feet) with a mean age of 48 years (range 12-81) [4]. The second study included 55 patients ( 72 feet) with a mean age of 56 years (range 24-81) [5]. In this case there are perhaps some patients who were included in both studies. However, in the study cohort from 1998 they reviewed the case records of 69 patients and, due to the deaths of unrelated causes of two patients (two feet), the lack of adequate follow-up from six patients (eight feet) and the exclusion of six patients with adolescent bunion or rheumatoid arthritis (six feet), 55 patients with 72 operated feet remained. This demonstrates that not the whole patient cohort from the former published study was included in the later study. Lee at al. published a prospective randomized study comparing two axial K-wires with a supplementary transverse $\mathrm{K}$-wire for proximal chevron osteotomy fixation [6]. The two cohorts contained 32 patients (41 feet) and 33 patients (44 feet), respectively. One year later Lee et al. published a

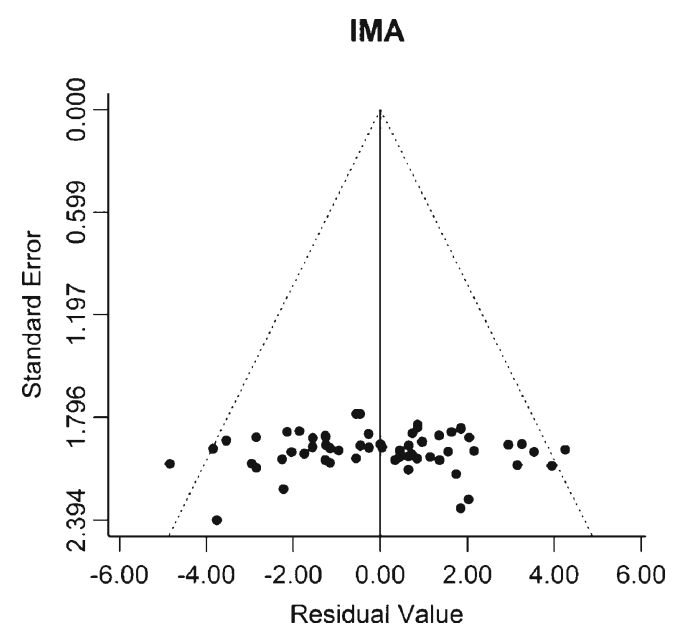

Fig. 1 Funnel plot for the delta intermetatarsal angle (IMA) metaanalysis 
HVA

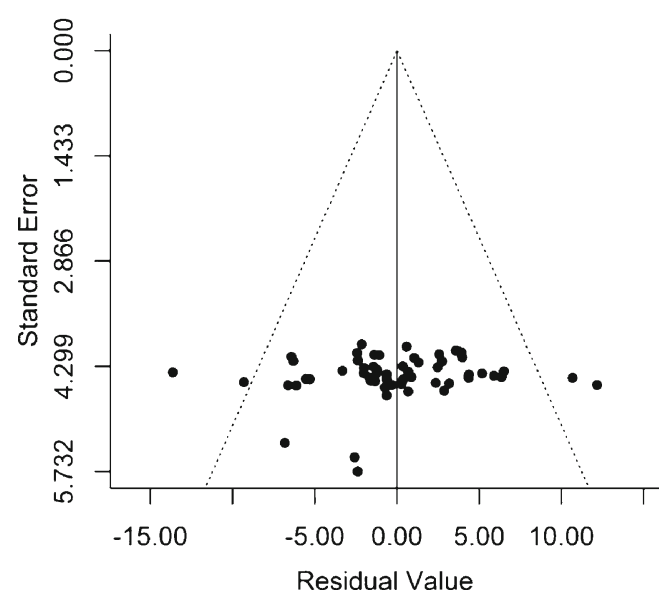

Fig. 2 Funnel plot for the delta hallux valgus angle (HVA) meta-analysis

retrospective comparative study with a patient group treated with unilateral and a group operated upon simultaneously for hallux valgus correction (52 patients, 69 feet) [7]. All patients in this study underwent the same surgical technique, in particular axial $\mathrm{K}$-wire fixation with two K-wires. The apprehension of He and Peng could indeed be correct in that we included some of Lee's patients in duplicate into meta-analysis.

2. Concerning the publication language, the authors did not restrict our systemic search to any languages. During the review process all publications revealed by the online databases MEDLINE, EMBASE, CINAHIL, Cochrane Central Register of Controlled Trials and Cochrane Database of Systematic Reviews, using the MeSH terms "(bunion OR hallux) AND (proximal OR crescentic OR basilar OR opening OR closing OR shelf OR ludloff) AND osteotomy" have been reviewed. Studies had to meet the inclusion criteria and a manual reference check of all accepted papers was performed to supplement the electronic search. Finally, only English- and Germanlanguage studies were eligible for meta-analysis
3. We would like to provide the funnel plots for delta IMA (Fig. 1) and delta HVA (Fig. 2). In consequence of a limitation in figure submission and publication the authors renounced the attachment of the drawn funnel plots in our original paper.

He and Peng raised some legitimate thoughts regarding our publication "Angular correction and complications of proximal first metatarsal osteotomies for hallux valgus deformity" [1]. After careful reflection we concluded that the study from Sammarco et al. [4] and the axial K-wire fixation group from Lee et al. [6] should be excluded from meta-analysis. As already mentioned, further high-quality prospective comparative studies are required and might change the observed effects.

\section{References}

1. Schuh R, Willegger M, Holinka J, Ristl R, Windhager R, Wanivenhaus AH (2013) Angular correction and complications of proximal first metatarsal osteotomies for hallux valgus deformity. Int Orthop 37(9): 1771-1780. doi:10.1007/s00264-013-2012-4

2. Coughlin MJ, Grimes S (2005) Proximal metatarsal osteotomy and distal soft tissue reconstruction as treatment for hallux valgus deformity. Keio J Med 54:60-65

3. Coughlin MJ, Jones CP (2007) Hallux valgus and first ray mobility. A prospective study. J Bone Joint Surg Am 89:1887-1898

4. Sammarco GJ, Brainard BJ, Sammarco VJ (1993) Bunion correction using proximal Chevron osteotomy. Foot Ankle 14:8-14

5. Sammarco GJ, Russo-Alesi FG (1998) Bunion correction using proximal chevron osteotomy: a single-incision technique. Foot Ankle Int 19:430-437

6. Lee K-B, Seo C-Y, Hur C-I, Moon E-S, Lee J-J (2008) Outcome of proximal chevron osteotomy for hallux valgus with and without transverse Kirschner wire fixation. Foot Ankle Int 29:1101-1106

7. Lee K-B, Hur C-I, Chung J-Y, Jung S-T (2009) Outcome of unilateral versus simultaneous correction for hallux valgus. Foot Ankle Int 30: $120-123$

8. Coughlin MJ, Roger A (1995) Mann Award. Juvenile hallux valgus: etiology and treatment. Foot Ankle Int 16:682-697 